\title{
Michel Offerlé, Les patrons des patrons. Histoire du
}

Medef

Paris, Odile Jacob, 2013

\section{Pierre Vernus}

\section{OpenEdition}

\section{Journals}

Édition électronique

URL : http://journals.openedition.org/travailemploi/6451

ISSN : 1775-416X

Éditeur

DARES - Ministère du Travail

\section{Édition imprimée}

Date de publication : 1 juillet 2014

Pagination : 108-110

ISSN : 0224-4365

\section{Référence électronique}

Pierre Vernus, « Michel Offerlé, Les patrons des patrons. Histoire du Medef », Travail et Emploi [En ligne],

139 | juillet-septembre 2014, mis en ligne le 01 juillet 2014, consulté le 22 septembre 2020. URL :

http://journals.openedition.org/travailemploi/6451

Ce document a été généré automatiquement le 22 septembre 2020.

(C) Direction de l'animation de la recherche, des études et des statistiques (Dares) 


\section{Michel Offerlé, Les patrons des patrons. Histoire du Medef}

Paris, Odile Jacob, 2013

\section{Pierre Vernus}

\section{RÉFÉRENCE}

Michel Offerlé, Les patrons des patrons. Histoire du Medef, Paris, Odile Jacob, 2013, 364 p.

1 Ce livre de Michel Offerlé constitue un excellent outil de décryptage des vicissitudes internes et des prises de position du Mouvement des entreprises de France (Medef). Bien plus, il fournira au lecteur des vues sur l'ensemble du champ de la représentation patronale ainsi qu'un important ensemble de données quantitatives et qualitatives, notamment de nombreux et intéressants extraits d'interviews.

2 Cette solide, et désormais incontournable, analyse sociologique de l'organisation faîtière patronale s'inscrit dans le prolongement des ouvrages antérieurs de l'auteur consacrés aux groupes de pression et aux organisations patronales. Elle participe au renouveau des recherches sur les organisations patronales qui s'observe depuis quelques années en France, renouveau auquel l'auteur a lui-même contribué par ses travaux et ceux qu'il a suscités.

3 Ce livre a pour ambition de « décrire et comprendre comment s'organisent et agissent collectivement ceux qu'on appelle couramment les patrons, stylisés collectivement comme le patronat » (p.10). Il se divise en cinq chapitres de longueur inégale, précédés d'une introduction dans laquelle M. Offerlé expose précisément sa démarche, les difficultés rencontrées lors de son enquête, sa problématique et les outils conceptuels qu'il a choisi de mobiliser et d'ajuster à cet objet d'étude particulier qu'est une confédération patronale.

4 Le premier chapitre analyse le cheminement qui a conduit à la refondation du Conseil national du patronat français (CNPF) qui apparaissait comme une structure devenue inadaptée, dépassée et représentant un patronat traditionnel et archaïque. La rupture 
s'est manifestée par l'adoption de nouveaux statuts, un changement de nom, qui entendait remplacer les représentations surannées du patronat et du conseil par les images plus proactives et dynamiques de l'entrepreneur, de l'entreprise et du mouvement, et, enfin, par un transfert du siège de l'organisation. Elle s'est accompagnée d'une modification des rapports de forces internes (contribution au financement, redécoupage des commissions, etc.), de l'intégration des nouvelles fédérations des services, de la substitution du principe majoritaire au consensus au sein du comité exécutif et d'une plus grande importance accordée aux structures territoriales. Ce dernier point n'est d'ailleurs pas sans rappeler la montée en puissance des associations régionales lors de la réforme de la $\mathrm{CGPF}^{1}$ en 1937. Le passage du CNPF au Medef s'est également traduit par le recours à des formes d'expression moins feutrées et plus directes incarnées par Ernest-Antoine Sellière et par une réorientation stratégique privilégiant l'économique sur le social, l'élargissement du domaine de la négociation autonome face à l'État et visant à remodeler profondément les paritarismes français instaurés après la seconde guerre mondiale. M. Offerlé souligne les résultats contrastés de ce changement de ton et de stratégie au regard de ses objectifs.

5 Le deuxième chapitre s'attache à situer le Medef dans la vaste nébuleuse des organismes qui prétendent porter la parole patronale et à démêler les structures confédérales dont la réforme de 1998 n'a effacé ni la complexité ni la labilité. Logiquement centré sur le Medef, le propos apporte néanmoins d'intéressants éclairages sur les autres organisations constitutives du noyau central de cette nébuleuse (CGPME, UPA, UNAPL ${ }^{2}$ ) ou sur d'autres structures (Afep ${ }^{3}$ ) généralement plus discrètes mais non moins influentes. Il rappelle opportunément l'ampleur de l'« hinterland ${ }^{4}$ organisationnel » développé par certaines organisations comme l'UIMM ${ }^{5}$ ou les opportunités offertes par les organismes paritaires dont l'étude reste encore largement à faire. Cette configuration feuilletée, entrecroisée et toujours mouvante s'explique en grande partie par la précédence historique des fédérations sur une confédération qui doit composer, parfois non sans difficultés, avec des identités sectorielles ou territoriales multiples. Les imbrications et les chevauchements qui caractérisent une telle structure font du Medef une organisation particulière pour laquelle il est difficile de déterminer des indicateurs de représentativité. Néanmoins, M. Offerlé montre que la confédération patronale est loin de rassembler l'ensemble des entreprises et ne reflète pas fidèlement la dynamique de l'économie française.

Le troisième chapitre s'intéresse aux représentants patronaux. Il dégage d'abord trois filières et trajectoires : en premier lieu les permanents qui mènent une carrière d' " apparatchiks"; en second lieu, la « filière militante » concernant soit de petits patrons dont l'investissement peut déboucher sur une carrière ascendante mais généralement limitée au niveau régional, soit des chefs d'entreprise ayant précocement adhéré à des mouvements de pensée et, enfin, dernière filière, celle des dirigeants d'entreprises sollicités en raison de leur visibilité sectorielle ou locale et dont l'investissement est temporaire ou sélectif. Dans un deuxième temps, il mobilise les outils de la sociologie des engagements pour éclairer la diversité des motivations qui conduisent les dirigeants patronaux à s'engager dans la vie de l'organisation. Enfin, d'une sociographie fondée sur quatre variables (sexe, âge, cursus de formation et type d'entreprise dirigée), se dégage l'image d'un groupe encore très masculin, âgé et beaucoup plus diplômé que la catégorie socioprofessionnelle qu'il représente, malgré une tendance à la féminisation et au rajeunissement. 
7 Le chapitre suivant s'attache à la manière dont les responsables gèrent la confédération, la représentent et font évoluer son rôle. On perçoit bien combien, le/la président/e doit composer avec l'histoire de la fonction, les modalités de sa désignation, le cloisonnement des structures de l'organisation, la grande autonomie des permanents ou le pouvoir des grandes fédérations. L'influence de ces dernières repose, avant tout, sur le montant de leurs contributions qui «mesurent moins la structuration de l'économie française projetée dans l'organisation que l'investissement que les grandes fédérations veulent engager dans l'interprofessionnel» (p. 264). Dans ce domaine, depuis 1999, si l'écart entre l'UIMM et les autres structures contributrices s'est réduit, la hiérarchie des principaux financeurs ne s'est pas modifiée.

Le dernier chapitre aborde la question du pouvoir du Medef en s'interrogeant dans un premier temps sur le périmètre légitime de son action, puis sur les ressources et les stratégies qu'il mobilise pour faire avancer les intérêts patronaux. L'auteur insiste sur la diversité des fonctions de l'organisation et des situations qu'elle doit affronter. Il rappelle, à juste titre, que ses interlocuteurs ne se réduisent pas aux syndicats de salariés et à l'État: le Medef s'adresse également aux organisations fédérales et locales, auxquelles il délivre un ensemble de services. Dans le même temps, son activité est complétée ou concurrencée par d'autres structures comme, par exemple l'Afep ou l'Institut de l'entreprise, alors que, à l'inverse, notamment sous la présidence de Laurence Parisot avant 2010, a pu se manifester une volonté d'intervention plus large dans tous les domaines constituant l'environnement de l'entreprise. Pour faire avancer ses idées, le Medef recourt à un répertoire d'actions spécifique : à la mobilisation par le nombre lors de regroupements sont préférés le communiqué de presse ou la déclaration et, surtout, le registre de l'expertise reposant sur l'usage du droit et de la statistique. Mais cette prétention à l'expertise doit constamment être réaffirmée et étayée car, dans la confrontation sociale, le discours patronal est perçu comme plus idéologique que neutre. Ce discours est le fruit de compromis reflétant les équilibres internes entre permanents et chefs d'entreprises élus - des compromis qui cherchent à dépasser les clivages entre petites et grandes entreprises ou entre les intérêts divergents des différents secteurs d'activité. Quels sont les effets de la mobilisation patronale ? Pour répondre à cette question, $\mathrm{M}$. Offerlé interroge la contribution du Medef à la définition des politiques publiques. Il fait ainsi remarquer que sur certaines questions, notamment fiscales, les représentants patronaux sont les seuls à intervenir, ce qui n'est pas le cas par exemple dans le domaine social. Le pouvoir d'influence du Medef est donc inégal selon les champs sociaux.

9 On l'aura compris ce livre rompt avec la représentation d'un patronat homogène et uni et d'un Medef qui, à la suite du CNPF, serait le pôle de coordination de l'influence patronale sur les différents centres de décision. Il en ressort plutôt l'image d'une organisation hétérogène, complexe, qui doit composer avec l'activité de ses fédérations et dont le discours doit tenter de synthétiser des intérêts divers et souvent divergents.

Bien sûr la lecture de ce livre suscite quelques regrets. Ainsi, dans le troisième chapitre, on aurait aimé disposer d'une pesée au moins grossière de l'importance des différents types de filières et de trajectoires des représentants patronaux. En outre, alors qu'à plusieurs reprises est rappelée la nécessité de prendre en compte le poids de l'histoire et la diversité des temporalités, on aurait souhaité que soit plus précisément évalué le rôle de la conjoncture politique dans la définition des stratégies d'influence. Mais n'estce pas le propre des livres pionniers de suggérer de nouvelles recherches? 


\section{NOTES}

1. En 1936 après la vague de grèves, la CGPF, fondée en 1919, modifie la signification de son sigle. La Confédération générale de la production française devient ainsi la Confédération générale du patronat français.

2. CGPME : Confédération générale du patronat des petites et moyennes entreprises. UPA : Union professionnelle artisanale. UNAPL : Union nationale des professions libérales.

3. Afep : Association française des entreprises privées.

4. Par hinterland, on entend l'ensemble des organisations liées ou dépendantes.

5. UIMM : Union des industries et des métiers de la métallurgie.

\section{AUTEURS}

\section{PIERRE VERNUS}

Université Lumière Lyon-2 - Laboratoire de recherche historique Rhône-Alpes (Larhra). 